Communication

ACS Publications

is published by the American Chemical Society. 1155 Sixteenth Street N.W., Washington, DC 20036

Published by American Chemical Society. Copyright @ American Chemical Society. However, no copyright claim is made to original U.S. Government works, or works produced by employees of any Commonwealth realm Crown government in the course of their duties. 
NANO

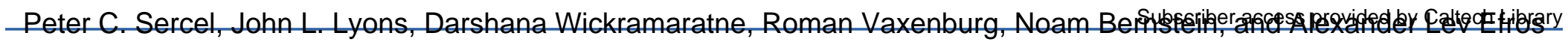

ACS Publications 


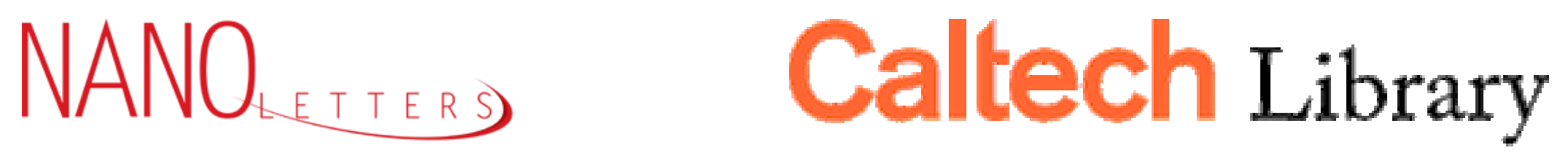

Subscriber access provided by Caltech Library "Just Accepted" manuscripts have been peer-reviewed and accepted for publication. They are posted online prior to technical editing, formatting for publication and author proofing. The American Chemical

ACS Publications 
NANO

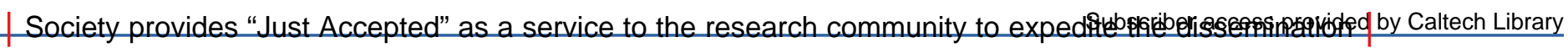
ACS Publications 
NANO

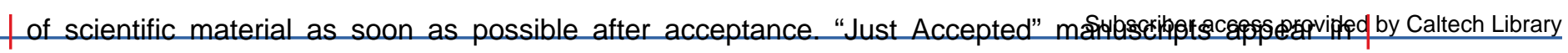
ACS Publications 


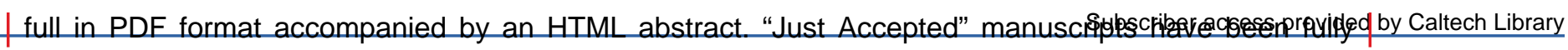

ACS Publications 


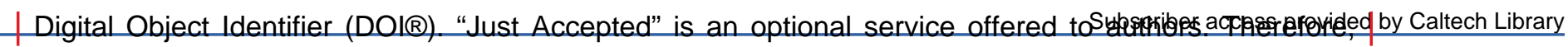

ACS Publications 


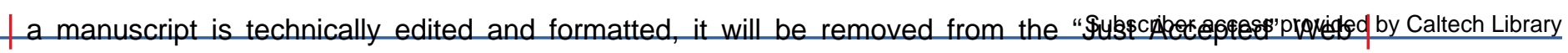

ACS Publications 
NANO

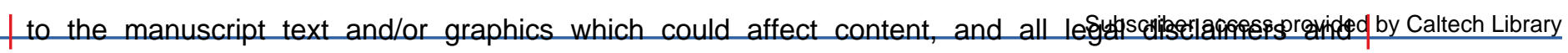
ACS Publications 
ethical guidelines that apply to the journal pertain. ACS cannot be held respoidsistitieforoccessontsvided by Caltech Library consequences arising from the use of information contained in these "Just Accepted" manuscripts.

ACS Publications 


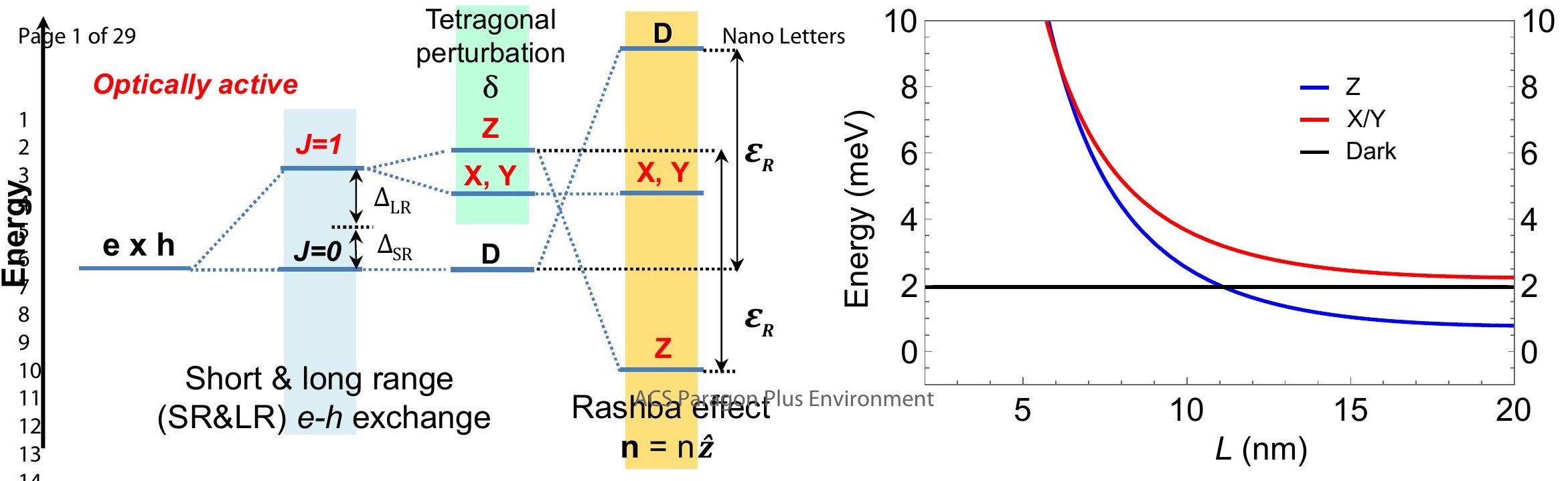




\title{
Exciton fine structure in perovskite
}

\section{nanocrystals}

\author{
Peter C. Sercel, ${ }^{*, \dagger}$ John L. Lyons, ${ }^{\ddagger}$ Darshana Wickramaratne, ${ }^{\ddagger}$ Roman \\ Vaxenburg, Noam Bernstein, ${ }^{\ddagger}$ and Alexander L. Efros*, \\ $\dagger$ Department of Applied Physics and Materials Science, California Institute of Technology, \\ Pasadena, California 91125, USA \\ $\ddagger$ Center for Computational Materials Science, U. S. Naval Research Laboratory, \\ Washington DC 20375, USA \\ \Computational Materials Science Center, George Mason University, Fairfax, Virginia \\ 22030, USA
}

E-mail: psercel@caltech.edu; efros@nrl.navy.mil 


\begin{abstract}
The bright emission observed in large size cesium lead halide perovskite nanocrystals (NCs) has recently been explained in terms of a bright exciton ground state [Becker et al., Nature 553, 189-193 (2018)], a claim which would make these materials the first known examples where the exciton ground state is not an optically forbidden dark exciton. This unprecedented claim has been the subject of intense experimental investigation which has so far failed to detect the dark ground state exciton. Here, we review the effective mass/electron-hole exchange theory for the exciton fine structure in cubic and tetragonal $\mathrm{CsPBBr}_{3} \mathrm{NCs}$. In our calculations the crystal field and the short-range electron-hole exchange constant were calculated using density functional theory, together with hybrid functionals and spin-orbit coupling. Corrections associated with long-range exchange and surface image charges were calculated using measured bulk effective mass and dielectric parameters. As expected, within the context of the exchange model, we find an optically inactive ground exciton level. However, in this model the level order for the optically active excitons in tetragonal $\mathrm{CsPbBr}_{3} \mathrm{NCs}$ is opposite to what has been observed experimentally. An alternate explanation for the observed bright exciton level order in $\mathrm{CsPBBr}_{3} \mathrm{NCs}$ is offered in terms of the Rashba effect, which supports the existence of a bright ground state exciton in these NCs. The size dependence of the exciton fine structure calculated for perovskite NCs shows that the bright-dark level inversion caused by the Rashba effect is suppressed by the enhanced electron-hole exchange interaction in small NCs.
\end{abstract}

\title{
Keywords
}

Exciton fine structure, lead halide perovskite, nanocrystals, electron-hole exchange, Rashba effect 
The optical band edge of all semiconductors ${ }^{2}$ or semiconductor nanostructures, including quantum wells ${ }^{3,4}$ quantum dots, ${ }^{5-7}$ nanocrystals $(\mathrm{NCs})^{8-13}$ nanorods $^{14}$ and nanoplatelets ${ }^{15}$ is characterized by multiple optical transitions. The degeneracies in the lowest levels are split into sublevels by the short- and long-range exchange interactions, spin-orbit coupling, intrinsic crystal field, and shape anisotropy, creating so-called exciton fine structure. The oscillator strengths of these sublevels can vary by many orders of magnitude, leading to optically active "bright" and inactive "dark" excitons, a dichotomy which holds in all nanostructures except those of the lowest possible symmetries. ${ }^{16}$

After more than 40 years of research, all observations of bulk semiconductors and heterostructures have shown a ground state exciton that was dark. For structures above the nm length scale, the dark-bright exciton splitting is usually smaller than the thermal energy, even at liquid-He temperatures, ${ }^{2-7}$ and the resulting photoluminescence (PL) decay rate is not strongly affected by temperature. For sufficiently small nanocrystals (NC), nanorods, and nanoplatelets, however, the splitting is of the order of tens of meV ${ }^{8-15}$ This leads to a particular temperature dependence of the PL: at low temperatures the ground state dominates, and the decay time is long, decreasing as the temperature increases and higher energy bright excitons are populated. ${ }^{15,17-20}$

The first measurements on colloidal lead-halide perovskite NCs a few years ago showed that this universally observed temperature dependence is not followed. ${ }^{21}$ Even without surface passivation they showed very bright PL, with room-temperature quantum yields of $50-90 \% .{ }^{22}$ This was traced to surprisingly short radiative lifetimes, ns-scale at room temperature, $20 \times$ faster than conventional semiconductor NCs (e.g. CdSe), and of order 250 ps at liquid-He temperatures, a thousand times shorter than conventional NCs. ${ }^{22}$ This reduction of lifetime with decreasing temperature suggested that the ground-state exciton in these 10$15 \mathrm{~nm}$ lead-halide perovskite NCs is bright, which would make them the first known example of such a system.

An intense search for a dark ground-state exciton in perovskite $\mathrm{NC}$ ensembles ${ }^{23-25}$ and 
single $\mathrm{NCs}^{26-28}$ at liquid-He temperatures did not show the line structure expected of such a dark exciton, i.e. a zero-phonon line and phonon-assisted satellites, as is typical for CdSe NCs. ${ }^{8-10}$ While it is possible that a bright higher-energy exciton has sufficiently efficient PL to compensate for the difference in thermal population in the case that the level spacing is comparable to the temperature, the dark exciton should still be observable under magnetic field, which mixes the different bright and dark states ${ }^{8,9}$ by an amount inversely proportional to the square of the splitting ${ }^{29}$ (the temperature and magnetic field dependence of the PL are presented in the Supporting Information). No lines corresponding to an activated dark exciton have been observed under a magnetic field. ${ }^{26,27}$ Instead, tetragonal crystal $\mathrm{CsPbBr}_{3}$ single NC samples show a low-lying singlet line, and an upper doublet line which is split by the magnetic field. ${ }^{26,28}$ Orthorhombic crystal NCs show only the three bright-exciton lines. $^{22}$ The observation of superradiance in ordered ensembles of perovskite $\mathrm{NCs}^{30}$ and coherent single photon emission in a single NC experiments ${ }^{31}$ are also consistent with the existence of a bright exciton ground state.

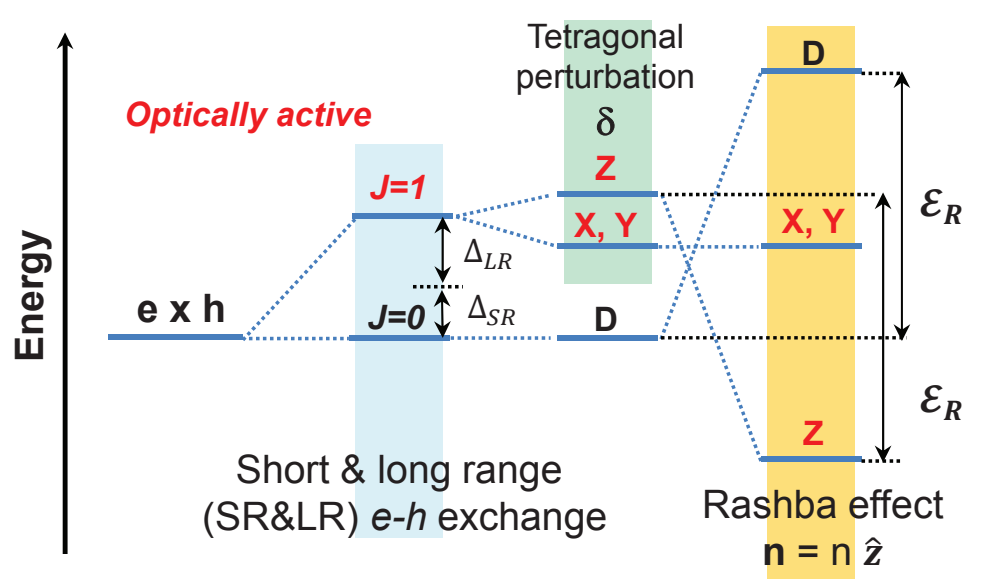

Figure 1: Cartoon showing fine structure of the exciton levels within the ground exciton manifold created by the short-range (SR) and long-range (LR) electron-hole exchange interaction, crystal field splitting, and Rashba splitting. The level structures are shown for a cube-shaped NC with tetragonal crystal structure with positive crystal field $\delta>0$. The right hand side of the figure shows the effect of Rashba splitting with a symmetry breaking axis along the $z$ direction, taken as the $c$ crystallographic axis, with splitting energy $\mathcal{E}_{R}$.

Another surprising feature of these NCs is that the splitting is of order $1 \mathrm{meV},{ }^{22,26,28}$ 
an order of magnitude larger than the values of the short-range (SR) exchange interaction splitting predicted using density functional theory (DFT). ${ }^{1}$ This can be explained by the Rashba effect (Figure 1), which also makes the bright exciton the lowest energy state ${ }^{1}$ and explains the failure so far to observe a magnetically activated dark exciton. Indeed, magnetooptical measurements of $\mathrm{CsPbBr}_{3}$ NCs show large Rashba terms. ${ }^{27}$ Others have suggested that the large splitting is connected with a combination of long-range (LR) exchange and shape anisotropy ${ }^{32,33}$ or an enhancement of exchange for small NCs combined with a giant crystal-field splitting. ${ }^{28}$ These size-dependent mechanisms for level splitting underscore the need for a model which spans the full size range, from the large size limit of weak confinement, to the small size, strong confinement limit.

In this letter we review the effective-mass/electron-hole exchange theory for the exciton fine structure in $\mathrm{CsPbBr}_{3} \mathrm{NCs}$ with cubic and tetragonal crystal symmetry. We compute the crystal field and SR exchange parameters for our model using DFT with hybrid functionals and spin-orbit coupling, and apply the model to determine the size-dependent exciton fine structure. As expected, without the Rashba effect we find an inactive ground-state exciton. Importantly, we find that in an exchange model, even including LR corrections, the calculated order for the optically active exciton levels in tetragonal $\mathrm{CsPbBr}_{3} \mathrm{NCs}$ is opposite to what has been observed experimentally. The Rashba effect, on the other hand, reverses the bright singlet/bright-doublet exciton level order, in addition to reversing the bright-dark level order in large NCs. We show that the bright-dark level inversion caused by the Rashba effect is suppressed by the enhanced electron-hole exchange interaction in small NCs.

Exciton fine structure. The short-range exchange interaction can be modelled as a contact interaction in terms of an effective spin operator acting on the electron and hole spins as, ${ }^{34}$

$$
\hat{H}_{\mathrm{exch}}^{S R}=\frac{1}{2} C \Omega\left[\mathbb{I}-\left(\boldsymbol{\sigma}_{e} \cdot \boldsymbol{\sigma}_{h}\right)\right] \delta\left(\boldsymbol{r}_{e}-\boldsymbol{r}_{h}\right) .
$$


In this expression, $\mathbb{I}$ is the $4 \times 4$ unit matrix, $\boldsymbol{\sigma}_{e}$ and $\boldsymbol{\sigma}_{h}$ are Pauli operators representing the electron and hole spin (not their total angular momenta), $\Omega$ is the volume of the crystal unit cell, $C$ is the exchange constant, defined in the Supporting Information in terms of the Bloch functions of the conduction and valence bands, and the delta function acts on the envelope functions. The exciton fine structure can be found after averaging eq 1 over the wave function of the excitons confined in the NCs.

In $\mathrm{CsPbBr}_{3}$ the conduction and valence band edges are two-fold degenerate. The total exciton wave function of a confined exciton can be written as a linear combination of the products of conduction $\left(\left|c_{1}\right\rangle\right.$ and $\left.\left|c_{2}\right\rangle\right)$ and valence $\left(\left|v_{1}\right\rangle\right.$ and $\left.\left|v_{2}\right\rangle\right)$ band edge Bloch functions multiplied by the envelope wave function of the confined exciton $f\left(\boldsymbol{r}_{e}, \boldsymbol{r}_{h}\right)$. The Bloch function of the valence band has $s$ symmetry and can be written as $\left|v_{1}\right\rangle=|S\rangle|\uparrow\rangle$ and $\left|v_{2}\right\rangle=|S\rangle|\downarrow\rangle$, where $|\uparrow\rangle,|\downarrow\rangle$ are the usual spin eigenstates. ${ }^{1}$ The Bloch functions of the conduction band edge depend strongly on the crystal symmetry and have been derived within an 8 -band $\boldsymbol{k} \cdot \boldsymbol{p}$ model, which takes into account the upper $J=3 / 2$ conduction band, and the tetragonal crystal field: 35,36

$$
\begin{aligned}
\left|c_{1}\right\rangle & =-\sin \theta|Z\rangle|\uparrow\rangle+\frac{-\cos \theta|X\rangle-i \cos \theta|Y\rangle}{\sqrt{2}}|\downarrow\rangle, \\
\left|c_{2}\right\rangle & =\frac{-\cos \theta|X\rangle+i \cos \theta|Y\rangle}{\sqrt{2}}|\uparrow\rangle+\sin \theta|Z\rangle|\downarrow\rangle .
\end{aligned}
$$

Here the terms $|X\rangle,|Y\rangle$, and $|Z\rangle$ denote orbital Bloch functions which transform as the functions having $p$ symmetry. The phase angle $\theta$ is defined via the spin-orbit splitting, $\Delta_{\mathrm{SO}}$, of the conduction band and the tetragonal crystal field parameter, $\delta$, as follows: ${ }^{35,36}$

$$
\tan 2 \theta=\frac{2 \sqrt{2} \Delta_{\mathrm{SO}}}{\Delta_{\mathrm{SO}}-3 \delta}, \quad \theta \leq \frac{\pi}{2}
$$

Note that in the case of cubic symmetry, $\delta=0$ so that $\tan 2 \theta=2 \sqrt{2}$ or $\theta \approx 35.26^{\circ}$. The parameters $\Delta_{\mathrm{SO}}$ and $\delta$ can in principle be derived from experiments or calculated using a 
higher theory. Results presented below use parameters calculated within DFT.

Averaging eq 1 over the four conduction and valence band Bloch function products, $\left|c_{1}\right\rangle\left|v_{1}\right\rangle,\left|c_{1}\right\rangle\left|v_{2}\right\rangle,\left|c_{2}\right\rangle\left|v_{1}\right\rangle$ and $\left|c_{2}\right\rangle\left|v_{2}\right\rangle$, we obtain the short-range (SR) exchange operator:

$$
\tilde{H}^{S R}=\Omega C \tilde{M}^{S R}(\theta) \delta\left(\boldsymbol{r}_{e}-\boldsymbol{r}_{h}\right)
$$

where the matrix $\tilde{M}^{S R}(\theta)$ that encodes the symmetry of the Bloch functions can be written,

$$
\tilde{M}^{S R}(\theta)=\left(\begin{array}{cccc}
\cos ^{2} \theta & 0 & 0 & 0 \\
0 & \sin ^{2} \theta & \sin ^{2} \theta & 0 \\
0 & \sin ^{2} \theta & \sin ^{2} \theta & 0 \\
0 & 0 & 0 & \cos ^{2} \theta
\end{array}\right) .
$$

Importantly, within the SR exchange interaction model the matrix $\tilde{M}^{S R}(\theta)$ completely determines the level order and the relative oscillator transition strengths of each level, independently of the envelope wave function of the confined exciton.

The size dependence of the exciton fine structure splitting can be obtained by averaging of eq 4 over the wave function of the confined exciton:

$$
\tilde{H}_{\mathrm{exch}}^{S R}=C \tilde{M}^{S R}(\theta) \Theta
$$

Here, $\Theta$ is the overlap factor taken over the volume $V$ of the cube-shaped NC, where $V=L^{3}$ for a NC of size $L$, representing the probability that the electron and hole reside in the same unit cell:

$$
\Theta=\Omega \iint_{V} d^{3} r_{e} d^{3} r_{h} f^{*}\left(\boldsymbol{r}_{e}, \boldsymbol{r}_{h}\right) \delta\left(\boldsymbol{r}_{e}-\boldsymbol{r}_{h}\right) f\left(\boldsymbol{r}_{e}, \boldsymbol{r}_{h}\right)=\Omega \int_{V} d^{3} r|f(\boldsymbol{r}, \boldsymbol{r})|^{2}
$$

Diagonalization of $\tilde{M}^{S R}$ for cubic perovskites yields a singlet level with eigenvalue 0 which corresponds to the dark exciton and 3 degenerate upper levels with eigenvalues equal to 
$2 / 3 C \Theta$ which are optically allowed. For tetragonal symmetry the 3 upper levels split, but the average of their eigenvalues remains $(2 / 3) C \Theta$.

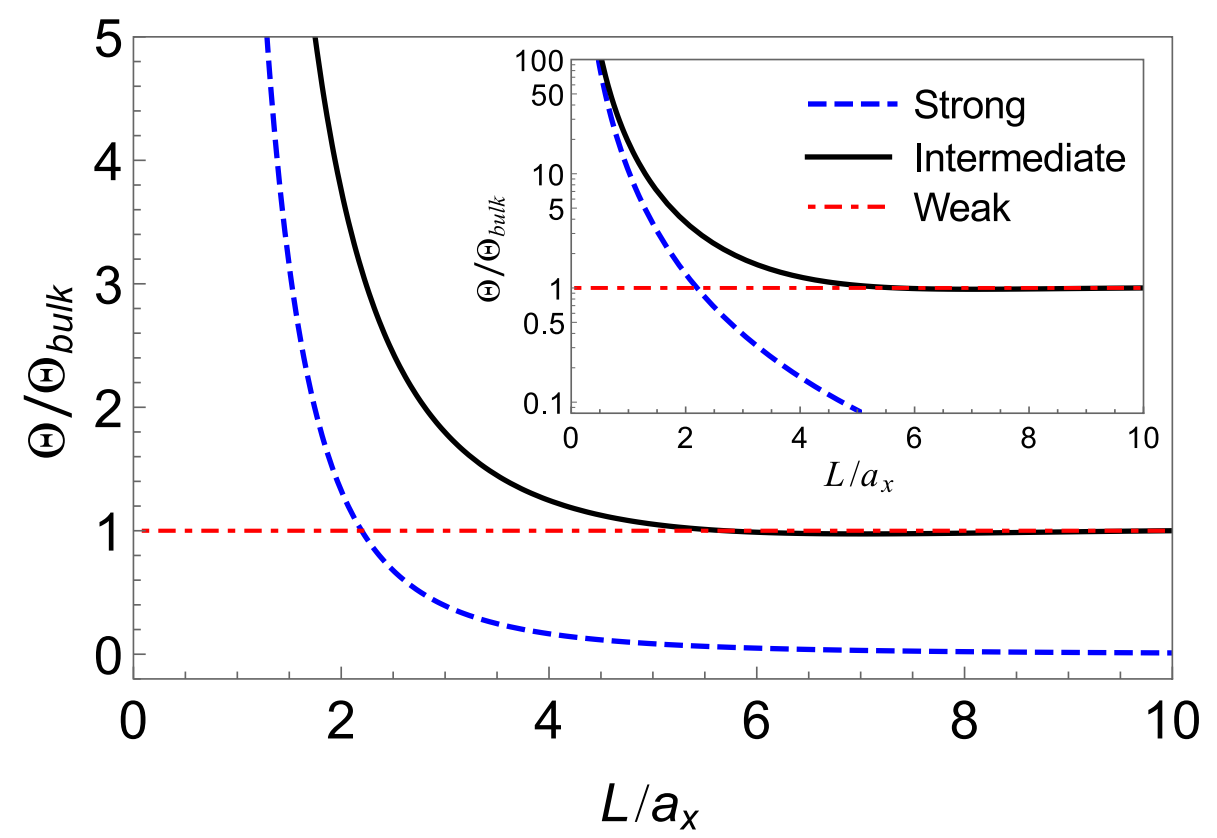

Figure 2: Exchange overlap factor, $\Theta$, versus NC size. $\Theta$ is given in terms of the bulk overlap factor, $\Theta_{b u l k}=\Omega /\left(\pi a_{x}^{3}\right)$, and is plotted versus the ratio $L / a_{x}$ of the edge length, $L$, of a cube-shaped $\mathrm{NC}$ to the exciton radius $a_{x}$. The solid black line represents the result of a variational calculation valid for the intermediate confinement regime, $L \sim a_{x}$. Also shown are the overlap factors calculated in the strong $\left(L<a_{x}\right.$, blue dashed line) and the weak ( $L \gg a_{x}$, red dashed line) confinement limits. Analytical expressions for the overlap factor in the various limits are summarized in Table 1 . The inset shows a log plot to facilitate examination of the small size limit.

Using the envelope function for the lowest energy exciton in the bulk, eq S8 of the Supporting Information, with hydrogenic function $\phi_{1 s}(\boldsymbol{r})=\exp \left(-r / a_{x}\right) / \sqrt{\pi a_{x}^{3}}$, where $a_{x}=$ $a_{0} \epsilon / \mu$ is the exciton Bohr radius, defined via the hydrogen Bohr radius, $a_{0}$, the reduced mass of the exciton $\mu$, and relative dielectric constant, $\epsilon$, the overlap factor can be found as $\Theta_{\text {bulk }}=\Omega /\left(\pi a_{x}^{3}\right)$. This allows elimination of exchange constant $C$ in favor of the measurable bulk singlet-triplet splitting, $\hbar \omega_{S T}=(2 / 3) C\left(\Omega / \pi a_{b}^{3}\right)$. We can then rewrite $\tilde{H}_{\text {exch }}^{S R}$ in eq 7 as,

$$
\tilde{H}_{\text {exch }}^{S R}=\frac{3}{2} \hbar \omega_{S T} \tilde{M}^{S R}(\theta)\left(\frac{\Theta}{\Theta_{\text {bulk }}}\right)
$$

The overlap function $\Theta$ was calculated using the envelope wave functions $f\left(\boldsymbol{r}_{e}, \boldsymbol{r}_{h}\right)$ found 
for the weak $\left(a_{x} \ll L\right)$, intermediate $\left(a_{x} \sim L\right)$ and strong $\left(a_{x} \gg L\right)$ confinement regimes (see Table S1 of the Supporting Information). The results of these calculations for all three regimes are summarized in Table 1. The size dependence of $\Theta(L)$ for all three regimes is shown in Figure 2. The figure shows that the intermediate confinement approximation for $\Theta(L)$ smoothly transfers into the weak confinement result in the limit of large size $\left(L \gg a_{x}\right)$ and into the strong confinement result in the limit $L<a_{x}$. This suggests that the expression for $\Theta(L)$ derived for the intermediate regime can be used for the entire size range.

Table 1: The exchange overlap factor, $\Theta$, and the SR and LR contributions to the average singlet-triplet splitting, $\overline{\Delta_{s t}^{S R}}$ and $\overline{\Delta_{s t}^{L R}}$, respectively, are shown for the three confinement regimes for a cube-shaped $\mathrm{NC}$ with edge length $L$. The $\mathrm{SR}$ contribution is given in terms of the bulk singlet-triplet splitting energy $\hbar \omega_{S T}$. The LR contribution, $\Delta_{s t}^{L R}$, is parameterized in terms of the bulk LT splitting energy, $\hbar \omega_{L T}$, given in the description of eq 14 . Normalization factor $N(\beta)$ is defined in eq S20 of the Supporting Information. Calculated values of the SR and LR exchange constants, $C^{S R}$ and $C^{L R}$, as well as $\hbar \omega_{S T}$ and $\hbar \omega_{L T}$ are given in Tables 3 and 4 .

\begin{tabular}{|l|c|c|c|}
\hline Regime & Overlap $\Theta$ & $\overline{\Delta_{s t}^{S R}}=2 / 3 C^{S R} \Theta$ & $\overline{\Delta_{s t}^{L R}}=2 / 3 C^{L R} \Theta$ \\
\hline \hline $\begin{array}{l}\text { Weak } \\
L \gg a_{x}\end{array}$ & $\Theta_{\text {Weak }}=\frac{\Omega}{\pi a_{x}^{3}}$ & $\hbar \omega_{S T}$ & $\frac{\hbar \omega_{L T}}{3}$ \\
\hline $\begin{array}{l}\text { Inter. } \\
L \sim a_{x}\end{array}$ & $\Theta_{\text {Inter }}=\frac{1}{N(\beta)} \frac{27}{8} \frac{\Omega}{L^{3}}$ & $\frac{1}{N(\beta)} \frac{27}{8} \pi\left(\frac{a_{x}}{L}\right)^{3} \hbar \omega_{S T}$ & $\frac{1}{N(\beta)} \frac{27}{8} \pi\left(\frac{a_{x}}{L}\right)^{3} \frac{\hbar \omega_{L T}}{3}$ \\
\hline $\begin{array}{l}\text { Strong } \\
L<a_{x}\end{array}$ & $\Theta_{\text {Strong }}=\frac{27}{8} \frac{\Omega}{L^{3}}$ & $\frac{27}{8} \pi\left(\frac{a_{x}}{L}\right)^{3} \hbar \omega_{S T}$ & $\frac{27}{8} \pi\left(\frac{a_{x}}{L}\right)^{3} \frac{\hbar \omega_{L T}}{3}$ \\
\hline \hline
\end{tabular}

Crystal field splitting of the triplet and dipole transition matrix elements. In NCs with the cubic lattice structure, the singlet-triplet splitting, $\Delta_{s t}$, completely describes the exciton fine structure (see Figure 1) because the triplet state is 3-fold degenerate. However, in tetragonal NCs the triplet degeneracy is broken as depicted in Figure 1. The splitting can be found by diagonalizing the matrix $\tilde{M}^{S R}$ in eq 5 for angles $\theta$ defined in eq 3 via a given set of spin-orbit coupling and crystal field parameters. The resulting energy can be 
expressed for each exciton eigenstate $X_{i}$, where $X_{i}=\{X, Y, Z, D\}$, in the general form,

$$
E_{X_{i}}(\theta)=C \Theta \tilde{f}_{X_{i}}(\theta)
$$

where the energy scale is set by $C \Theta$, and $\tilde{f}_{X_{i}}(\theta)$ is a dimensionless eigenvalue of the matrix $\tilde{M}^{S R}$. The functions $\tilde{f}_{X_{i}}$ are straightforward to evaluate; the results are given in Table 2 for the cubic and tetragonal phases. The average of $\tilde{f}_{X_{i}}(\theta)$ taken over the 3 triplet states $X, Y, Z$ is $2 / 3$ so that the average singlet-triplet splitting, $\overline{\Delta_{s t}^{S R}}$, in all cases equals $2 / 3 C \Theta$. The transition dipole matrix elements (DMEs) are found as the matrix element of the moTable 2: Dimensionless energy, $\tilde{f}_{X_{i}}(\theta)$, and the dimensionless transition dipole matrix elements (DMEs) $\tilde{\boldsymbol{p}}_{X_{i}}$ for each exciton eigenstate $X_{i}$ in perovskite NCs with cubic and tetragonal crystal structures. The $\tilde{\boldsymbol{p}}_{X_{i}}$ are expressed in the vector format $\left\{\tilde{p}_{x}, \tilde{p}_{y}, \tilde{p}_{z}\right\}$ where the $x, y, z$ axes are aligned to the $\mathbf{a}, \mathbf{b}, \mathbf{c}$ axes of the respective unit cell. The order of levels in the table is shown for a positive tetragonal crystal field $\delta$.

\begin{tabular}{||c||c|c||l|l||}
\hline \multicolumn{1}{||c||}{} & \multicolumn{3}{|c||}{ Cubic } & \multicolumn{2}{c||}{ Tetragonal } \\
\hline $\begin{array}{c}\text { State } \\
\left(X_{i}\right)\end{array}$ & $\begin{array}{l}\text { Energy } \\
\left(\tilde{f}_{X_{i}}\right)\end{array}$ & $\begin{array}{c}\text { DME } \\
\left(\tilde{\boldsymbol{p}}_{X_{i}}\right)\end{array}$ & $\begin{array}{l}\text { Energy } \\
\left(\tilde{f}_{X_{i}}\right)\end{array}$ & $\begin{array}{l}\text { DME } \\
\left(\tilde{\boldsymbol{p}}_{X_{i}}\right)\end{array}$ \\
\hline \hline $\mathrm{Z}$ & $\frac{2}{3}$ & $\sqrt{\frac{2}{3}}\{0,0,1\}$ & $2 \sin ^{2} \theta$ & $\{0,0, \sqrt{2} \sin \theta\}$ \\
\hline $\mathrm{Y}$ & $\frac{2}{3}$ & $\sqrt{\frac{2}{3}}\{0, i, 0\}$ & $\cos ^{2} \theta$ & $i\{0, \cos \theta, 0\}$ \\
\hline $\mathrm{X}$ & $\frac{2}{3}$ & $\sqrt{\frac{2}{3}}\{1,0,0\}$ & $\cos ^{2} \theta$ & $\{\cos \theta, 0,0\}$ \\
\hline \hline $\begin{array}{l}\text { Triplet } \\
\text { Ave } \tilde{f}\end{array}$ & $\frac{2}{3}$ & $\mathrm{NA}$ & $\frac{2}{3}$ & $\mathrm{NA}$ \\
\hline \hline Dark & 0 & $\{0,0,0\}$ & 0 & $\{0,0,0\}$ \\
\hline \hline
\end{tabular}

mentum operator, $\hat{\boldsymbol{p}}$, between the exciton eigenfunction, $\psi_{X_{i}}$, and the crystal ground state $G$. Denoting the exciton DME as $\boldsymbol{P}_{X_{i}}$ one can write,

$$
\boldsymbol{P}_{X_{i}}=\left\langle\psi_{X_{i}}|\hat{\boldsymbol{p}}| G\right\rangle=\boldsymbol{p}_{X_{i}} \iint_{V} f\left(\boldsymbol{r}_{e}, \boldsymbol{r}_{h}\right) \delta\left(\boldsymbol{r}_{e}-\boldsymbol{r}_{h}\right) d^{3} r_{e} d^{3} r_{h}
$$


where $\boldsymbol{p}_{X_{i}}=\left\langle\Phi_{X_{i}}|\hat{\boldsymbol{p}}| G\right\rangle$, and within the effective mass approximation the exciton wave function $\psi_{X_{i}}=\Phi_{X_{i}} f\left(\boldsymbol{r}_{e}, \boldsymbol{r}_{h}\right)$ is expressed in terms of the product of the conduction and valence band Bloch functions (denoted here as $\Phi_{X_{i}}$ ) and the smooth envelope function, $f\left(\boldsymbol{r}_{e}, \boldsymbol{r}_{h}\right)$. Eq 10 contains an overlap integral between the electron and hole wave functions, which we will label $\mathcal{O} \equiv \iint_{V} f\left(\boldsymbol{r}_{e}, \boldsymbol{r}_{h}\right) \delta\left(\boldsymbol{r}_{e}-\boldsymbol{r}_{h}\right) d^{3} r_{e} d^{3} r_{h}=\int_{V} f(\boldsymbol{r}, \boldsymbol{r}) d^{3} r$, taken over the entire NC volume $V$. The result can be expressed for each exciton eigenstate $X_{i}$, in the general form,

$$
\boldsymbol{P}_{X_{i}}(\theta)=\mathcal{O} \boldsymbol{p}_{X_{i}}(\theta)=\mathcal{O} P_{c v} \tilde{\boldsymbol{p}}_{X_{i}}(\theta)
$$

where $P_{c v}=\langle S|\hat{\boldsymbol{P}}| Z\rangle$ is the Kane matrix element and $\tilde{\boldsymbol{p}}_{X_{i}}(\theta)$ is a dimensionless matrix element that depends on the crystal structure parameters and has vectorial character. The analytical expressions for $\tilde{\boldsymbol{p}}_{X_{i}}(\theta)$ for NCs with different crystal symmetries are shown in Table 2. Using the DME from eq 11 one can calculate the oscillator strength for each exciton state $X_{i}$ as: $f_{X_{i}}=2 \boldsymbol{P}_{X_{i}}^{2} /\left(\hbar \omega m_{0}\right)$, where $\hbar \omega$ is the transition energy and $m_{0}$ is the free electron mass. This expression can be rewritten as:

$$
f_{X_{i}}=\mathcal{O}^{2} f_{0} \tilde{f}_{X_{i}}(\theta)
$$

where $f_{0}=E_{p} / \hbar \omega$ is approximately equal to the ratio of the Kane energy $E_{p}=2 P_{c v}^{2} / m_{0}$ to the band gap $E_{g}$, and we define $\tilde{f}_{X_{i}}=\left|\tilde{\boldsymbol{p}}_{X_{i}}(\theta)\right|^{2}$ as the reduced oscillator strength, the quantity that reflects the crystal symmetry. The functions $\tilde{f}_{X_{i}}$ describe all dependences of the oscillator strength on the light polarization. One can check by direct calculation that these are the same functions as were found in the expression for the energy, eq 9 and in Table 2 , where $\tilde{f}_{i}(\theta)$ and $\tilde{\boldsymbol{p}}_{X_{i}}(\theta)$ are given for each of the exciton exchange eigenstates $X, Y, Z, D$ for the cubic and tetragonal phases. The factor $\mathcal{O}^{2}$ in eq 12 is responsible for the giant oscillator transition strength phenomenon predicted in ref 37, which has been demonstrated experimentally and theoretically described in $\mathrm{CsPbX}_{3} \mathrm{NCs}^{1}$ 
Long range exchange corrections. In the discussion above, we neglect completely the effect of the LR exchange interaction, which in bulk semiconductors causes a splitting between the longitudinal and transverse optically active exciton states. ${ }^{34,38-40}$ The consideration of this phenomenon in the Supporting Information is made using the approach developed originally for bound excitons by Bir and Pikus. ${ }^{41}$ In the basis of the exciton states $X_{i}$ (where $X_{i}=\{X, Y, Z, D\}$ ) which diagonalize the SR exchange interaction, the Hamiltonian describing the LR exchange interaction in cube-shaped NCs can be written:

$$
\tilde{H}_{X_{i}}^{L R}=\frac{4 \pi}{3} \frac{e^{2} \hbar^{2}}{\epsilon_{\infty} m_{0}^{2} E_{g}^{2}}\left|P_{c v}\right|^{2} \tilde{f}_{X_{i}} \frac{\Theta}{\Omega} .
$$

Here $\epsilon_{\infty}$ is the high frequency dielectric constant, the definitions given in eqs 11 and 12 are used for the dipole matrix elements, and the orthogonality of the DMEs is utilized for $\boldsymbol{p}_{X_{i}}$. We can simplify eq 13 , re-writing it via the longitudinal-transverse splitting of the exciton, $\hbar \omega_{L T}$,

$$
\tilde{H}_{X_{i}}^{L R}=\frac{\hbar \omega_{L T}}{2} \tilde{f}_{X_{i}} \frac{\pi a_{x}^{3}}{\Omega} \Theta
$$

where $\hbar \omega_{L T}=\left(8 / 3 \epsilon_{\infty} a_{x}^{3}\right)\left(\hbar e P_{c v} / m_{0} E_{g}\right)^{2}$ in simple parabolic band semiconductors such as the perovskites. ${ }^{38,41-43}$ Since the average value of $\tilde{f}_{X_{i}}$ taken over the triplet $X, Y, Z$ states is $\overline{\tilde{f}_{X_{i}}}=2 / 3$, the average LR exchange splitting between the ground state singlet and the upper triplet states is

$$
\overline{\Delta_{s t}^{L R}} \equiv \frac{2}{3} C^{L R} \Theta=\frac{\hbar \omega_{L T}}{3} \cdot \frac{\Theta}{\Theta_{\text {bulk }}},
$$

where $C^{L R} \equiv\left(\hbar \omega_{L T} / 2\right)\left(\pi a_{x}^{3} / \Omega\right)$ represents the LR exchange contribution to the exchange constant. Expressions for the LR contribution to the average singlet-triplet exchange splitting, $\overline{\Delta_{s t}^{L R}}$, are given for each confinement regime alongside the short-range contribution, $\overline{\Delta_{s t}^{S R}}$, in Table 1. 
The analysis of the LR exchange interaction in NCs highlights an important conceptual difficulty discussed in the literature since 1993. The LR contribution to the exchange energy derived here within the $\boldsymbol{k} \cdot \boldsymbol{p}$ model originates solely due to the contact $\left(\sim \delta\left(\boldsymbol{r}_{e}-\boldsymbol{r}_{h}\right)\right)$ electron-hole interaction (see eq S44 in the Supporting Information) and, as such, can be interpreted as a self-interaction of the exciton polarization within the same unit cell. In 1993, Takagahara analyzed the LR exchange in spherical NCs, Ref. [44], explicitly excluding this contact interaction, resulting in a vanishing of the LR exchange correction in spherical or cube-shaped NCs of semiconductors with simple bands. This question was revisited by Goupalov and Ivchenko in Ref. [45] within the framework of an empirical tight-binding model. They concluded that the LR exchange corrections in NCs should be modified by the inclusion of a correction factor, $\gamma$, where $\gamma \rightarrow 1$ in the case that the band-edge optical transitions are principally inter-atomic, while $\gamma \rightarrow 0$ in the case that the band edge transition is strictly intra-atomic. The inter-atomic case, involving transitions between two different atoms in the unit cell, is realized in III-V or II-VI semiconductors such as CdSe. ${ }^{45,47,48}$ In the case that the band-edge optical transitions are principally intra-atomic, the LR exchange correction vanishes in this model, consistent with the analysis of Takagahara for spherical or cube shaped NCs. ${ }^{44}$ This situation may apply for perovskite $\mathrm{ABX}_{3}$ semiconductors, where the large band edge oscillator transition strength is connected with the allowed intra-atomic $s$ - to $p$ - transition on the same B atom. ${ }^{46}$ In this case, the LR correction described by eq 15 should be characterized by a $\gamma$-dependent $C^{L R}(\gamma):{ }^{45}$

$$
C^{L R}(\gamma)=\gamma \frac{\hbar \omega_{L T}}{2} \frac{\pi a_{x}^{3}}{\Omega}
$$

where $0<\gamma<1$. The value of $\gamma$, which reflects the extent to which LR exchange contributes to the exchange splitting in perovskite NCs, remains to be established; we treat this term as a phenomenological parameter in our model.

Effect of dielectric discontinuity at the nanocrystal surface. In semiconductor 

2

NCs embedded in a dissimilar dielectric medium, the electron-hole pair interaction is not a simple distance-dependent Coulomb interaction $\sim e^{2} /\left|\boldsymbol{r}_{e}-\boldsymbol{r}_{h}\right|$ as was assumed in eq S41 of the Supporting Information. This is due to the electron and hole each interacting with the image charges of the opposite carrier, which arise due to the dielectric discontinuity at the NC surface. To estimate the impact of this phenomenon, we examine the magnitude of these corrections in a spherical NC, considered as an approximation to the corrections in a cube-shaped NC. In the Supporting Information, we show that the correction to the singlet-triplet splitting in the weak confinement regime is,

$$
\Delta_{s t}^{\text {diel }}=\frac{4}{\pi^{2}}\left(\frac{\kappa-1}{\kappa+2}\right) \hbar \omega_{L T}
$$

where $\kappa=\epsilon_{i} / \epsilon_{o}$ is the ratio of the dielectric constants inside $\left(\epsilon_{i}\right)$ and outside $\left(\epsilon_{o}\right)$ of the NC. This result was previously derived using an electrodynamic approach by Ekimov et al. ${ }^{49}$ Using eq 17, we incorporate the effect of the dielectric discontinuity as a correction to the exchange constant including the factor $\gamma$ to modify the LR exchange splitting parameter $\hbar \omega_{L T}$ as before:

$$
C^{\text {diel }}=\gamma \hbar \omega_{L T} \frac{6}{\pi^{2}}\left(\frac{\kappa-1}{\kappa+2}\right) \frac{\pi a_{x}^{3}}{\Omega}
$$

This procedure is correct in the weak confinement limit $L \gg a_{x}$, but overestimates the dielectric correction in the strong confinement regime $\left(L \ll a_{x}\right)$; for a spherical $\mathrm{NC}$ the error in the strong confinement limit is $17 \%$ for $\kappa=3.3$.

Rashba effect. In addition to the electron-hole exchange, it has been shown that Rashba terms in perovskite NCs also affect the exciton fine structure. ${ }^{1,27}$ We will focus on NCs with tetragonal crystal symmetry and an inversion symmetry-breaking direction $v_{z}$ along the $z$ axis taken as the tetragonal $c$-axis. In that case the Rashba Hamiltonian $H_{R}^{e(h)}$ for electrons (holes) can be written as: ${ }^{1} H_{R}^{e(h)}=\alpha_{e(h)} v_{z}\left(\sigma_{x}^{e(h)} \hat{p}_{y}^{e(h)}-\sigma_{y}^{e(h)} \hat{p}_{x}^{e(h)}\right) / \hbar$, where $\alpha_{e}$ and $\alpha_{h}$ are the Rashba coefficients for the electron and hole, respectively. The Rashba terms do not affect 
the exciton fine structure in the strong confinement limit where electron and hole motion are uncorrelated; however, in the weak confinement limit, they lead to exciton fine structure splitting which can be found in second order perturbation theory. In NCs having tetragonal crystal symmetry, the exciton fine structure correction is described as: ${ }^{1}$

$$
\Delta E^{R}=-2\left(A_{c}-A_{r}\right) \mathcal{E}_{R}\left(\boldsymbol{\sigma}_{x}^{e} \sigma_{x}^{h}+\sigma_{y}^{e} \sigma_{y}^{h}\right)
$$

where $A_{c}=128 /\left(27 \pi^{2}\right)$ and $A_{r}=(64 / 81 \sqrt{3})^{2}$ are coefficients reflecting the center-of-mass and relative motion of the exciton, and $\mathcal{E}_{R}=\alpha_{e} \alpha_{h} \mu / \hbar^{2}$ is the exciton Rashba energy.

Calculation of the exciton fine structure. We have evaluated three contributions to the electron-hole exchange splitting in cube shaped NCs: The SR exchange interaction, the LR exchange interaction associated with the electron-hole contact interaction, and the exchange corrections associated with the dielectric discontinuity between NCs and their surrounding medium. The total exchange constant can be written as the sum of these three contributions: $C^{t o t}=C^{S R}+C^{L R}+C^{d i e l}$, resulting in the exciton fine structure described by the following expression:

$$
E_{X_{i}}(\theta)=C^{t o t} \Theta \tilde{f}_{X_{i}}(\theta)
$$

which replaces eq 9 derived for the short-range exchange interaction. The crystal-structuredependent dimensionless functions $\tilde{f}_{X_{i}}(\theta)$ given in Table 2 are unchanged.

In order to calculate the size-dependent exchange splitting using eq 20, one needs the exchange constants and the crystal field. To access the SR exchange constant and the crystal field, we build up the SR exchange Hamiltonian using hybrid DFT ${ }^{50,51}$ as implemented in the VASP $\operatorname{code}^{52}$ using a basis of the band-edge DFT wave functions for cubic and tetragonal $\mathrm{CsPbBr}_{3}$ (see eq S36-S37). These calculations yielded lattice parameters and energy gaps that are in good agreement with experiment ${ }^{53,54}$ (Table S2 in the Supporting Information).

Diagonalization of the exchange Hamiltonian enables determination of the bulk SR ex- 
Table 3: Short-range exchange constants, exciton energies, oscillator strengths, and crystal field parameters for cubic and tetragonal $\mathrm{CsPBr}_{3}$ calculated using DFT with hybrid functionals. The SR exchange constant $C^{S R}$ for each phase is determined by diagonalization of the SR exchange Hamiltonian, described in eq S36 of the Supporting Information, using band-edge wavefunctions from the DFT calculation. $C^{S R}$ scales as the inverse of the unit cell volume, $\Omega$, which is also given for each phase. The quasi-cubic exchange constant $C_{q c}^{S R}$, which is scaled by the ratio of the unit cell volume to the cubic cell volume, is also given. The dimensionless energy of each exciton state, $\tilde{E}_{X_{i}}=E_{X_{i}} / C^{S R} \Theta$, is measured relative to the singlet state energy defined as the zero of energy $\left(E_{D}=0\right)$. Also given is the reduced oscillator strength, $\tilde{f}_{i}$ (see eq 12). The $\boldsymbol{x}, \boldsymbol{y}, \boldsymbol{z}$ axes are defined as the $\mathbf{a}, \mathbf{b}, \mathbf{c}$ axes of the unit cell. In $\boldsymbol{k} \cdot \boldsymbol{p}$ theory, $\tilde{E}_{X_{i}}=\tilde{f}_{X_{i}}$. The phase parameter $\theta$ (see eq 2 ) is determined both from the DFT-calculated reduced energies and from the reduced oscillator strengths, using the expressions in Table 2. The corresponding crystal field values, determined from eq 3 with $\mathrm{SO}$ parameter $\Delta_{\mathrm{SO}}=1.54 \mathrm{eV}$ from DFT, are also given.

\begin{tabular}{|c|c|c|c|c|}
\hline & \multicolumn{2}{|c|}{ Cubic } & \multicolumn{2}{|c|}{ Tetragonal } \\
\hline$C^{S R}$ & \multicolumn{2}{|c|}{$C_{c u b}^{S R}=509 \mathrm{meV}$} & \multicolumn{2}{|c|}{$C_{t e t}^{S R}=219 \mathrm{meV}$} \\
\hline Cell Vol. $\Omega$ & \multicolumn{2}{|c|}{$\Omega_{c u b}=0.2104 \mathrm{~nm}^{3}$} & \multicolumn{2}{|c|}{$\Omega_{t e t}=0.4127 \mathrm{~nm}^{3}$} \\
\hline$C_{q c}^{S R}$ & \multicolumn{2}{|c|}{$C_{c u b}^{S R}=509 \mathrm{meV}$} & \multicolumn{2}{|c|}{$C_{q c}^{S R}=429 \mathrm{meV}$} \\
\hline State $\left(X_{i}\right)$ & $\tilde{E}_{X_{i}}$ & $\tilde{f}_{X_{i}}$ & $\tilde{E}_{X_{i}}$ & $\tilde{f}_{X_{i}}$ \\
\hline Z & 0.667 & 0.667 & 0.759 & 0.765 \\
\hline Y & 0.667 & 0.667 & 0.620 & 0.617 \\
\hline $\mathrm{X}$ & 0.667 & 0.667 & 0.620 & 0.617 \\
\hline $\begin{array}{l}\text { Triplet } \\
\text { Ave }\end{array}$ & 0.667 & 0.667 & 0.667 & 0.667 \\
\hline Dark & 0 & 0 & 0 & 0 \\
\hline $\begin{array}{l}\text { Tetr. phase } \theta \\
\text { Tetr. CF } \delta\end{array}$ & & $264^{\circ}$ & $\begin{array}{l}38.037^{\circ} \\
+153 \mathrm{meV}\end{array}$ & $\begin{array}{l}39.483^{\circ} \\
+226 \mathrm{meV}\end{array}$ \\
\hline
\end{tabular}


change constant $\left(C^{S R}\right)$ and the crystal field. The results of this calculation are shown in Table 3 for cubic and tetragonal $\mathrm{CsPbBr}_{3}$. Since the exchange constant scales as the inverse of the unit cell volume, it is useful to compare quasi-cubic values $\left(C_{q c}^{S R}\right)$ which are scaled by the ratio of the unit cell volumes; this parameter is somewhat lower in the tetragonal phase than for the cubic phase. In the table, the energy of each exciton state, $E_{X_{i}}$, is given with the singlet state energy defined as the zero of energy. Table 3 also shows the calculated reduced oscillator strengths, $\tilde{f}_{X_{i}}$ (defined in eq 12 ). In the 8 -band $\boldsymbol{k} \cdot \boldsymbol{p}$ theory, the exciton energy $E_{X_{i}}=C \Theta \tilde{f}_{X_{i}}$ of the $X_{i}$ exciton is also proportional to $\tilde{f}_{X_{i}}$. Remarkably, inspection of Table 3 shows that the relationship between $\tilde{f}_{X_{i}}$ and $E_{X_{i}}$ obtained by DFT closely follows the $\boldsymbol{k} \cdot \boldsymbol{p}$ prediction. The average of the quantity $\tilde{f}_{X_{i}}$ over the triplet states $X, Y, Z$ is $2 / 3$ for all phases as shown in the table in agreement with the $\boldsymbol{k} \cdot \boldsymbol{p}$ theory, but the crystal field splits the triplet in the tetragonal phase. The crystal field parameter $\delta$, determined from the exciton fine structure energies found using DFT, is shown in Table 3. To determine it we used $\Delta_{\mathrm{SO}}=1.54 \mathrm{eV}$ from DFT, using the connection between the ratio of the $Z$ and $X$ or $Y$ exciton fine structure energies and the crystal field phase angle $\theta$ is determined by, $E_{Z} / E_{X, Y}=2 \tan ^{2} \theta$, and used eq 3. Importantly, the crystal field was not determined from the upper conduction band splitting as suggested in Refs.[28,35,36]. This splitting is strongly influenced by higher band effects not included in an 8-band $\boldsymbol{k} \cdot \boldsymbol{p}$ framework, as described below.

A key result is that for the tetragonal phase, the SR exchange interaction places the $Z$ exciton above the degenerate $X / Y$ doublet as a result of a positive tetragonal crystal field, $\delta>0$. The positive tetragonal crystal field, $\delta>0$ is in agreement with DFT calculations performed on inorganic tin halide perovskites of tetragonal structure. ${ }^{46}$ The previous analysis of the exciton fine structure in tetragonal hybrid organic perovskites ${ }^{36}$ assumed a negative crystal field based on measurements of $2-\mathrm{D}$ hybrid organic perovskites. ${ }^{55}$ This assumption led to the prediction that the $Z$ exciton lies below the degenerate $X / Y$ doublet. Inclusion of LR exchange and dielectric corrections does not change this level order in cube shaped NCs 

calculated by diagonalization of the SR exchange Hamiltonian described by eq S36 in the Supporting Information, using band-edge functions calculated for tetragonal $\mathrm{CsPbBr}_{3}$ using DFT with hybrid functionals. The value reported in the table is in reference to the quasicubic cell, with cell volume $\Omega$ set equal to that of the cubic phase. The LR exchange constant is calculated using bulk energy band parameters of $\mathrm{CsPbBr}_{3}$ at $\mathrm{T}=2 \mathrm{~K}$ reported in ref [56]. The dielectric correction term is calculated for $\kappa=3.29$ corresponding to NCs embedded in a dielectric medium with refractive index 1.49. For easy reference, the right two columns show the impact of each term on the singlet-triplet splitting in the weak confinement size limit, $L \gg a_{x}$.

\begin{tabular}{||l|c|c||l|c||}
\hline Contribution & Parameter & Value & $\begin{array}{l}\text { Weak confinement } \\
\Delta E_{s t}\left(L \gg a_{x}\right)\end{array}$ & Value \\
\hline \hline SR exchange & $C_{q c}^{S R}$ & $429 \mathrm{meV}$ & $\Delta E_{s t}^{S R}=\hbar \omega_{s t}$ & $0.661 \mathrm{meV}$ \\
\hline $\begin{array}{l}\text { LR exchange } \\
C_{q c}^{L R}\end{array}$ & $760 \mathrm{meV}$ & $\Delta E_{s t}^{L R}=\hbar \omega_{L T} / 3$ & $1.174 \mathrm{meV}$ \\
\hline $\begin{array}{l}\text { Dielectric } \\
\text { correction, } \\
\kappa=3.29\end{array}$ & $C_{q c}^{\text {diel }}$ & $400 \mathrm{meV}$ & $\Delta E_{s t}^{\text {diel }}$ & $0.617 \mathrm{meV}$ \\
\hline \hline Total & $C_{q c}^{\text {tot }}$ & $1589 \mathrm{meV}$ & $\Delta E_{s t}^{\text {tot }}$ & $2.452 \mathrm{meV}$ \\
\hline \hline
\end{tabular}

Figure 3 shows the size dependence of the exciton fine structure levels in cube-shaped tetragonal $\mathrm{CsPbBr}_{3}$ NCs. Panels (a) and (b) show results accounting for electron-hole exchange only, with different assumed values of the LR exchange factor $\gamma$ in panel (a) and various $\kappa$ responsible for the magnitude of the dielectric correction in panel (b). Parameters used in the calculation are summarized in Table 4. One can see that the level order is the same for all values of $\gamma$ and $\kappa$, because in the exchange model the level order is determined 
only by the crystal field. Notably, the order of the bright $\mathrm{Z}$ and $\mathrm{X} / \mathrm{Y}$ exciton levels calculated here in (a) and (b) is opposite to the level order within the experimental exciton fine structure observed in $\mathrm{CsPbBr}_{3} \mathrm{NCs}^{26,28}$
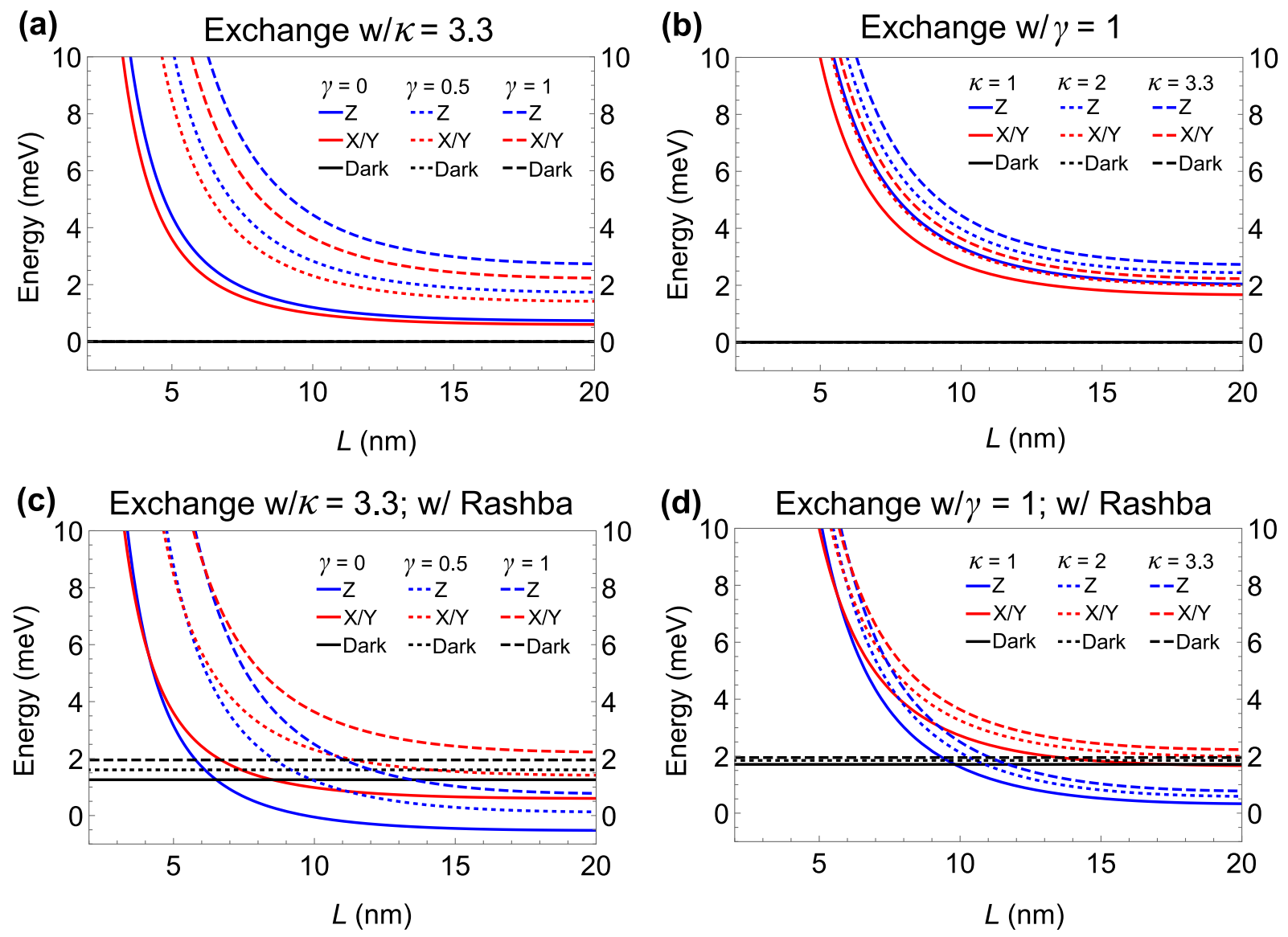

Figure 3: Exciton fine structure of tetragonal $\mathrm{CsPbBr}_{3} \mathrm{NCs}$ versus NC size. Plots show fine structure energy plotted versus edge length $L$ of a cube-shaped NC. Panels (a) and (b) show results accounting for electron-hole exchange only, with LR exchange factor $\gamma=0,0.5$ and 1 , with $\kappa=3.3$ in panel (a) and $\gamma=1$ with $\kappa=1,2$, and 3.3 in panel (b). The fine structure splitting is controlled only by the SR exchange interaction if $\gamma=0$, while LR exchange corrections are included completely if $\gamma=1$. With $\kappa=1$ dielectric corrections vanish, while $\kappa=3.3$ corresponds to a $\mathrm{NC}$ embedded in a medium of refractive index $n=1.49$. Exchange constants used in the calculation are summarized in Table 4. In panels (c) and (d), the exciton fine structure is shown with the addition of a phenomenological Rashba term with an exciton Rashba energy $\mathcal{E}_{R}$ for each set of parameters set to reproduce the experimental 1 $\mathrm{meV}$ splitting between the $\mathrm{Z}$ and $\mathrm{X} / \mathrm{Y}$ excitons at a NC size of $9 \mathrm{~nm} .{ }^{26}$ The factors $\gamma$ and $\kappa$ are the same between panels (c) and (a), and between (d) and (b).

In Figure 3 panels (c) and (d) we include the Rashba term, described by eq 19, in addition to electron-hole exchange. The factors $\gamma$ and $\kappa$ are the same between panels (c) and (a), and 

in each case to produce a splitting between the $Z$ exciton and the $X / Y$ doublet of $1 \mathrm{meV}$ at a size $L=9 \mathrm{~nm}$, as measured experimentally on tetragonal $\mathrm{CsPbBr}_{3} \mathrm{NCs}$ by $\mathrm{Fu}$ et al. ${ }^{26}$ This requires values $\mathcal{E}_{R}=1.15,1.47,1.79 \mathrm{meV}$ in panel (c), with $\gamma=0,0.5,1$, respectively, and $\mathcal{E}_{R}=1.57,1.70,1.79 \mathrm{meV}$ in panel (d), where $\kappa=1,2,3.3$ respectively. The value of $\mathcal{E}_{R}$ increases with increasing $\gamma$ and $\kappa$, since an increase of these terms increases the exchange splitting. These numbers are somewhat larger than the estimate made in ref [1], which neglected completely the effect of exchange in the estimation based on the level splitting.

\section{Discussion of available experimental data. Comparison of the two upper} and lower panels in Figure 3 clearly shows that inclusion of the Rashba term is required to match the experimentally observed $Z$ and $X / Y$ level order over a large range of sizes. The level order of the dark singlet state and the optically active $\mathrm{Z}$ state is also inverted relative to the situation with no Rashba term. As a result, in large NCs the ground state exciton is dipole active. ${ }^{1}$ However, since the exchange splitting increases as $1 / L^{3}$, as the size of the $\mathrm{NC}$ is reduced there will be a crossover below which the level order switches, and the dark exciton becomes the ground state. Figure 3 shows that the crossover size depends on $\gamma$ and $\kappa$. The predicted exciton ground state found in these calculations is consistent with the fast emission experimentally measured in NCs of size range $10-15 \mathrm{~nm}$.

Figure 3 shows also that the splitting between the bright fine structure levels is essentially independent of the NC size for $L$ larger than $\sim 10 \mathrm{~nm}$, a phenomenon which is connected with the size independence of the exchange splitting in the weak confinement limit $\left(a_{x} \ll L\right)$. In small size NCs, the exchange interaction increases the splitting between the bright exciton levels (see Figure $3 \mathrm{a}, \mathrm{b}$ ). The presence of the Rashba terms, however, results in a reduction of the splitting between the bright exciton levels with decreasing $\mathrm{NC}$ size (see Figure $3 \mathrm{~d}, \mathrm{c}$ ). This feature of the calculation agrees with the experimental size-dependent fine structure splitting of Ramade et al. ${ }^{28}$ reportedly in the $\sim 7 \mathrm{~nm}$ size range.

In the Supporting Information we consider the effect of the NC shape anisotropy on the 
exciton fine structure splitting, a mechanism proposed in Refs. 32,33. Using the parameters in Tables 3 and 4 and neglecting intrinsic crystal field effects we find that for a $20 \%$ uniaxial shape anisotropy ${ }^{57}$ in a $9 \mathrm{~nm} \mathrm{NC}$ the expected splitting $E_{X / Y}-E_{Z}$ is $0.7 \mathrm{meV}$ for $\gamma=1$. Including the tetragonal crystal field in the calculations makes the $E_{X / Y}-E_{Z}$ splitting negative. The size dependence of the splitting is shown in Figure S-7 of the Supporting Information. Importantly, for an asymmetric shaped $\mathrm{NC}$ of fixed aspect ratio, the fine structure splitting increases with decreasing size, a feature which can be used to distinguish this mechanism from fine structure splitting due to the Rashba effect.

The relationship between the oscillator transition strengths and the fine structure splitting calculated using DFT is in reasonable agreement with eq 20. The DFT calculation yields a fine structure ratio $\tilde{E}_{Z} / \tilde{E}_{X / Y}$ somewhat smaller than the ratio of oscillator strengths $\tilde{f}_{Z} / \tilde{f}_{X / Y}$. However, the $\boldsymbol{k} \cdot \boldsymbol{p}$ model completely fails to describe the relationship between the crystal field and the upper conduction band energies. In the 8-band $\boldsymbol{k} \cdot \boldsymbol{p}$ model, the tetragonal crystal field splits the upper $J=3 / 2$ conduction bands by an amount, ${ }^{26,28,35,36}$

$$
\Delta_{J=3 / 2}=\frac{\Delta_{s o}+\delta}{2}-\frac{1}{2} \sqrt{\Delta_{s o}^{2}-\frac{2}{3} \Delta_{s o} \delta+\delta^{2}} .
$$

In fact, using eq 21 to determine the crystal field $\delta$ from the upper $J=3 / 2 \mathrm{CB}$ splitting within the 8 -band $\boldsymbol{k} \cdot \boldsymbol{p}$ expressions ${ }^{28,35,36}$ causes an overestimation of the crystal field and therefore the fine structure splitting by approximately $3 \mathrm{x}$. The error is connected with the presence of higher conduction bands not included in the 8-band $\boldsymbol{k} \cdot \boldsymbol{p}$ model that significantly increase the upper conduction band splitting, but have only a small, second-order influence on the exciton fine structure splitting.

This is demonstrated in Figure 4 which shows the band edges of lowest 8 conduction bands calculated in DFT for a structural interpolation sequence starting with $\mathrm{CsPbBr}_{3}$ in the cubic structure and ending in the tetragonal structure. In addition to the 6 conduction bands included in the standard $\boldsymbol{k} \cdot \boldsymbol{p}$ analysis, which are folded onto the Z-point of the tetragonal 
Brillouin zone (BZ) from the R-point of the cubic structure, there is an additional 2-fold degenerate band $382 \mathrm{meV}$ above the four $J=3 / 2$ states which originates from the z-directed $\mathrm{X}$-point of the BZ of the cubic structure. As the tetragonal distortion increases, this state couples with the R-point derived states. The resulting level repulsion increases the upper $J=3 / 2$ conduction band splitting by a nearly a factor of $\sim 3$ above what is predicted in the 8 -band $\boldsymbol{k} \cdot \boldsymbol{p}$ theory. ${ }^{28,35,36}$ Figure 4 shows the excellent quantitative agreement between DFT calculations and results from a 14-band $\boldsymbol{k} \cdot \boldsymbol{p}$ model, which will be described elsewhere.

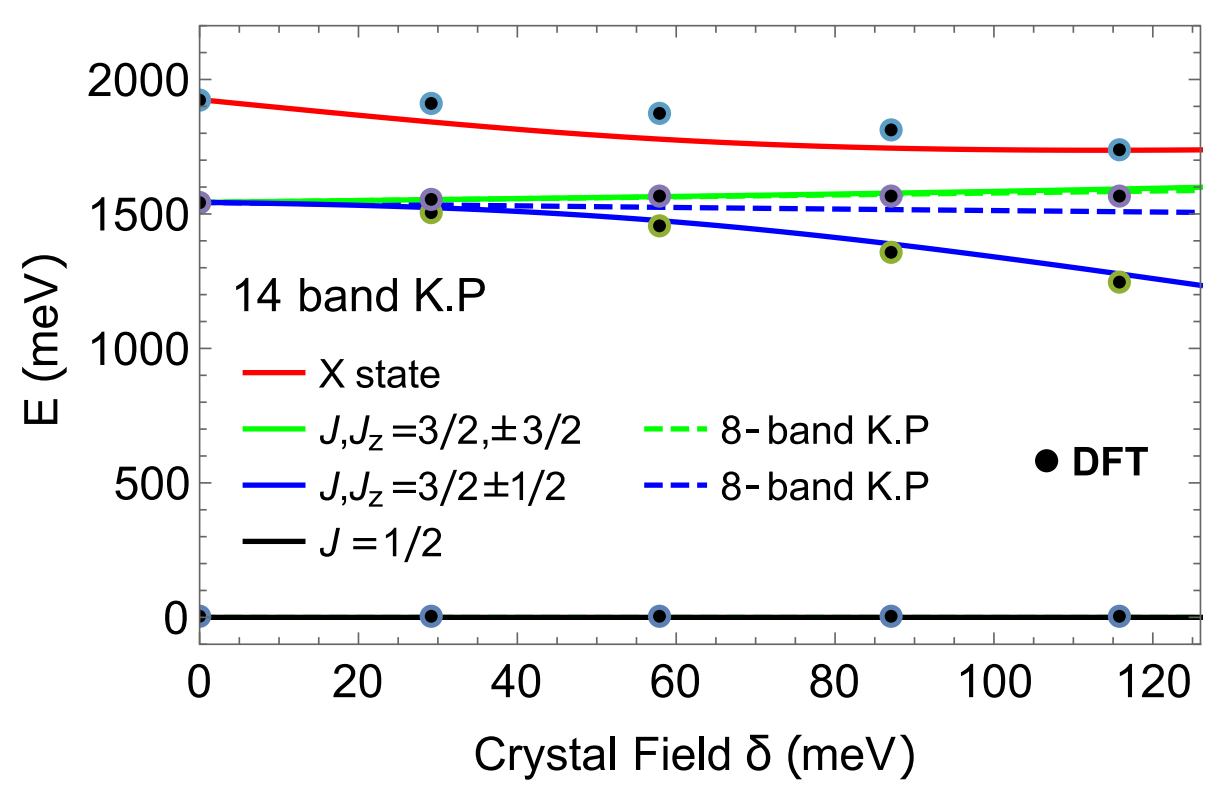

Figure 4: Effect of tetragonal crystal field on upper conduction band splitting in $\mathrm{CsPbBr}_{3}$. A linear structural interpolation sequence starting with $\mathrm{CsPbBr}_{3}$ in the cubic structure and ending in the tetragonal structure was calculated by DFT using hybrid functionals. The figure shows the upper conduction bands calculated within DFT (black points) relative to the lowest conduction band, plotted as a function of the tetragonal crystal field, $\delta$, determined by parameter fitting to a 14 -band $\boldsymbol{k} \cdot \boldsymbol{p}$ model. The solid lines show the energies of the upper X-derived (red) and $J=3 / 2$ conduction bands (green, $J_{z}= \pm 3 / 2$, blue, $J_{z}= \pm 1 / 2$ ) calculated in the 14 -band $\boldsymbol{k} \cdot \boldsymbol{p}$ model. The dashed lines show the upper $J=3 / 2$ levels calculated within the 8 -band $\boldsymbol{k} \cdot \boldsymbol{p}$ model using the same crystal field parameters.

In summary, a complete theory of the exciton fine structure in $\mathrm{CsPbBr}_{3} \mathrm{NCs}$ has been developed, taking into account the lattice distortion that transforms the cubic to the tetragonal perovskite phase, as well as the electron-hole exchange interaction in these materials. The 
crystal field parameters and the SR electron-hole exchange constant for tetragonal $\mathrm{CsPbBr}_{3}$ were calculated using DFT. Corrections to the electron-hole exchange energy associated with LR exchange and surface image charges were calculated using measured bulk effective mass and dielectric parameters. We demonstrated that the experimentally observed bright exciton level order in tetragonal $\mathrm{CsPbBr}_{3} \mathrm{NCs}$ can be explained in terms of the Rashba effect, which provides additional support for the existence of a bright ground state exciton in these NCs. It was shown, however, that the bright-dark level order inverts in small NCs, which consequently have a dark exciton ground state.

Acknowledgments. P.C.S. acknowledges support from the Center for Hybrid Organic Inorganic Semiconductors for Energy (CHOISE) an Energy Frontier Research Center funded by the Office of Basic Energy Sciences, Office of Science within the US Department of Energy. J.L., D.W., R.V., N.B. and Al L.E. acknowledge support from the U. S. Office of Naval Research through the U. S. Naval Research Laboratory's core research program. The work of J.L.L. and Al.L.E was supported by the Laboratory-University Collaboration Initiative of the DoD Basic Research Office. D.W. acknowledges support from the National Research Council fellowship at the US Naval Research Laboratory.

The authors declare no competing financial interests.

Supporting Information Available: Rate equation analysis of PL and magneto-PL for $\mathrm{CsPbBr}_{3} \mathrm{NCs}_{\mathrm{P}}$ assuming a slowly emitting dark ground state. Band edge exciton wave functions and energies. DFT calculation of $\mathrm{CsPbBr}_{3}$ band structure. Calculation of SR and LR exchange and corrections to the exchange splitting due to image charge effects and NC shape anisotropy.

\section{References}

(1) Becker, M. A.; Vaxenburg, R.; Nedelcu, G.; Sercel, P. C.; Shabaev, A.; Mehl, M. J.; Michopoulos, J. G.; Lambrakos, S. G.; Bernstein, N.; Lyons, J. L.; Stiferle, T.; Mahrt, 
R. F.; Kovalenko, M. V.; Norris, D. J.; Raino, G.; Efros, Al. L. Nature 2018, 553, 189 $-193$.

(2) Knox, R.S., Theory of excitons Academic Press, New York and London, 1963.

(3) Jeukens, C. R. L. P. N.; Christianen, P. C. M.; Maan, J. C.; Yakovlev, D. R.; Ossau, W.; Kochereshko, V. P.; Wojtowicz, T.; Karczewski, G.; Kossut, Phys. Rev. B 2002, $66,235318$.

(4) Kusrayev, Yu. G.; Zakharchenya, B. P.; Karczewski, G.; Wojtowicz, T.; Kossut, J. Solid State Commun. 1997, 104, 465-468.

(5) Bayer, M.; Ortner, G.; Stern, O.; Kuther, A.; Gorbunov, A. A.; Forchel,A.; Hawrylak, P.; Fafard, S.; Hinzer, K.; Reinecke, T. L.; Walck, S. N.; Reithmaier, J. P.; Klopf, F.; Schaäfer, F. Phys. Rev. B 2002, 65, 195315.

(6) Smoleński, T.; Kazimierczuk, T.; Goryca, M.; Jakubczyk, T.; Kłopotowski, Ł.; Cywiński, Ł.; Wojnar, P.; Golnik, A.; Kossacki, P. Phys. Rev. B. 2012 86, 241305(R).

(7) Korkusinski M.; Hawrylak, P.; Phys. Rev. B. 2013, 87, 115310.

(8) Nirmal, M. D.; Norris, J.; Kuno, M.; Bawendi, M. G.; Efros, Al. L.; Rosen, M. Phys. Rev. Lett. 1995, 75, 3728-3731.

(9) Efros, Al. L.; Rosen, M.; Kuno, M.; Nirmal, M.; Norris, D. J; \& Bawendi, M. G. Phys. Rev $B$ 1996, 54, 4843-4856.

(10) Chamarro, M.; Gourdon, C.; Lavallard, P.; Lublinskaya O.; Ekimov, A. I. Phys. Rev B. 1996 53, 1336-1342.

(11) Efros, Al. L. Semiconductor and Metal Nanocrystals: Synthesis and Electronic and Optical Properties edited by Klimov, V. I., Ch. 3, p. 103 Marcel Dekker, New York, 2003.

(12) Califano, M.; Franceschetti, A; Zunger, A. Phys. Rev. B 2007, 75, 115401. 
(13) Korkusinski, M.; Voznyy, O; Hawrylak, P. Phys. Rev. B 2010, 82, 245304.

(14) Shabaev A.; Efros, AL. L. Nano Lett. 2004, 4, 1821-1825.

(15) Biadala, L.; Liu, F.; Tessier, M. D.; Yakovlev, D. R.; Dubertret, B.; Bayer, M. Nano Lett. 2014, 14, 1134-1139.

(16) Sercel, P. C.; Shabaev, A.; Efros, Al. L. Nano Lett. 2017, 17, 4820-4830.

(17) Labeau, O.; Tamarat, P.; Lounis, B. Phys. Rev. Lett. 2003, 90, 257404.

(18) Biadala, L.; Louyer, Y.; Tamarat, P.; Lounis, B. Phys. Rev. Lett. 2009, $103,037404$.

(19) Biadala, L.; Louyer, Y.; Tamarat, P.; Lounis, B. Phys. Rev. Lett 2010, 105, 157402.

(20) Biadala, L.; Siebers, B.; Beyazit, Y.; Tessier, M. D.; Dupont, D.; Hens, Z.; Yakovlev, D. R.; and Bayer, M. ACS Nano 2016, 10, 3356-3364.

(21) Protesescu, L.; Yakunin,S.; Bodnarchuk,M.I.; Krieg, F.; Caputo,R.; Hendon, C.H.; Yang, R. X.; Walsh, A.; and Kovalenko, M. V., Nano Lett. 2015 15, 3692-3696.

(22) Rainò, G.; Nedelcu, G.; Protesescu, L.; Bodnarchuk, M. I.; Kovalenko, M. V.; Mahrt, R. F.; and Stoferle, T., ACS Nano 2016, 10, 2485-2490.

(23) Canneson, D.; Shornikova, E.V.; Yakovlev,D.R.; Rogge, T.; Mitioglu, A.A.; Ballottin, M.V.; Christianen, P.C.M.; Lhuillier, E.; Bayer, M. and Biadala, L. Nano Lett. 2017, $17,6177-6183$.

(24) Chen, L.; Li, B.; Zhang, C.; Huang, X.; Wang, X.; and Xiao, M. Nano Lett. 2017, 18, 2074-2080.

(25) Xu, K.; Vliem, J. F.; and Meijerink, A. J. Phys. Chem. C 2019, 123, 979 - 984.

(26) Fu, M.; Tamarat, P.; Huang,H.; Even, J.; Rogach, A.L.; and Lounis, B., Nano Lett. 2017 17, 2895-2901. 
(27) Isarov, M.; Tan, L. Z.; Bodnarchuk, M. I.; Kovalenko, M. V.; Rappe, A. M.; Lifshitz, E., Nano Lett. 2017 ,17, 5020-5026.

(28) Ramade, J.; Andriambariarijaona, L.M.; Steinmetz, V.; Goubet, N.; Legranda, L.; Barisien,T.; Bernardot,F.; Testelin, C.; Lhuillier, E.; Bramati, A.; Chamarro, M. Nanoscale 2018, 10, 6393-6401.

(29) Rodina, A. V.; Efros, Al. L. Phys. Rev. B. 2016, 93, 155427.

(30) Rainó, G.; Becker M.A.; Bodnarchuk, M. I.; Mahrt, R. F.; Kovalenko, M. V.; and Stöferle, T., Nature 2018, 563, 671-675.

(31) Utzat, H.; Sun, W.; Kaplan, A. E. K.; Krieg, F.; Ginterseder, M.; Spokoyny, B.; Klein, N. D.; Shulenberger, K.E.; Perkinson, C. F.; Kovalenko, M. V.; and Bawendi, M. G. Science 2019, 363, 1068-1072.

(32) Nestoklon, M.O.; Goupalov,S.V.; Dzhioev,R.I.; Ken, O.S.; Korenev,V.L.; Kusrayev, Yu.G.; Sapega,V.F.; de Weerd; Gomez, L.; Gregorkiewicz, T. ; Lin,J.; Suenaga,K.; Fujiwara,Y.; Matyushkin, L.B.; and Yassievich,I.N. Phys. Rev. B 2018, 97, 235304.

(33) Ben Aich, R.; Saidi, I.; Ben Radhia, S.; Boujdaria, K.: Barisien, T.; Legrand, L.; Bernardot, F.; Chamarro, M.; and Testelin, C. Phys. Rev. Appl 2019, 11, 034042.

(34) Rossler, U.; Trebin, H.-R. Phys. Rev. B 1981, 23, 1961-1970.

(35) Nagamune, Y. ; Takeyama, S.; and Miura,N., Phys. Rev. B 1991, 43, 12401-12405.

(36) Yu, Z. G. Sci. Rep., 2016, 6, 28576.

(37) Rashba, E. I. \& Gurgenishvili, G. E. Sov. Phys. Solid State 1962, 4, 759-760 .

(38) Pikus, G. E.; Bir, G. L. Zh. Eksp. Teor. Fiz. 1971, 60, 195-208.[Soviet Physics JETP 197133 108-114]. 
(39) Bir, G. L. and Pikus, G. E. Symmetry and strain-induced effects in semiconductors, Wiley (1974).

(40) Denisov, M. M. and Makarov, V. P., Phys. Stat. Sol. B 1973 56 9-58.

(41) Pikus, G. E.; Bir, G. L. Zh. Eksp. Teor. Fiz. 1972, 62, 324-332; [Soviet Physics JETP 1972, 35, 174-178].

(42) Goupalov, S. V.; Ivchenko. E. L. J. Cryst. Growth 1998, 184/185, 393 -397; Acta Physica Polonica 1998, A94, 341-346.

(43) Goupalov, S. V.; Ivchenko. E. L. Fiz. Tverd. Tela 2000, 42, 1976 -1984; [Phys. Sol. Stat. 2000 42, 2030-2038].

(44) Takagahara. T. Phys. Rev. B. 1993, 47, 4569 -4584.

(45) Goupalov, S. V.; Ivchenko. E. L. Fiz. Tverd. Tela 2001, 43, 1791-1798 . [Phys. Sol. Stat. 2001, 43, 1867-1875].

(46) Huang, L.-Y.; and Lambrecht, W. R. L, Phys. Rev. B. 2013, 88, 165203.

(47) Sercel, P. C.; Efros, Al. L., Nano Lett.2018, 18, 4061-4068.

(48) Franceschetti, A.; Wang, L. W.; Fu, H.; Zunger, A. Phys. Rev. B., 1998 58, 13367-13370 $(\mathrm{R})$.

(49) Ekimov, A. I.; Onushchenko, A. A.; Raikh, M. E.; Efros, Al. L., Zh. Eksp. Teor. Fiz. 1986, 90, 1795-1807; [ Sov. Phys. JETP 1986, 63, 1054-1060].

(50) Kohn, W.; and Sham, L.J., Phys. Rev. 1965, 140, A1133-A1138.

(51) Heyd, J.; Scuseria, G. E.; and Ernzerhof, M., J. Chem. Phys. 2003, 118, 8207-8215.

(52) Kresse, G., and Furthmüller, J. Phys. Rev. B 1996, 54, 11169-11186. 
(53) Stoumpos, C. C.; Malliakas, C. D.; Peters, J. A.; Liu, Z.; Sebastian, M.; Im, J.; Chasapis, T. C.; Wibowo, A. C.; Chung, D. Y.; Freeman, A. J.; Wessels, B. W.; and Kanatzidis, M. G. Crystal Growth \& Design 2013, 13, 2722-2727.

(54) Pazhuk, I. P.; Pydzirailo, N. S.; and Matsko, M. G. Sov. Phys. Sol. State 1981, 23, 1263-1265.

(55) Hirasawa, M., Ishihara, T.; Goto, T. J. Phys. Soc. Jpn 1994, 63, 3870-3879.

(56) Yang, Z.; Surrente, A.; Galkowski, K.; Miyata, A.; Portugall, O.; Sutton, R. J.; Haghighirad, A. A.; Snaith, H. J.; Maude, D. K.; Plochocka, P.; and Nicholas, R. J. ACS Energy Lett. 2017, 2, 1621-1627.

(57) Bertolotti, F.; Protesescu, L.; Kovalenko, M.V.; Yakunin, S.; Cervellino, A.; Billinge, S.J.L.; Terban, M.W.; Pedersen, J.S.; Masciocchi, N. and Guagliardi, A., ACS Nano 2017, 11, 3819-3831. 